\title{
Heartbeat: Reporting guidelines for high quality clinical cardiology research
}

Publication in a reputable journal is an essential step in clinical cardiovascular research. Publication allows both dissemination and archiving of research results, including details of methodology, so that other researchers and the general public can assess the reliability of the data presented. In addition to selecting the best research papers for publication, the editorial team of each journal helps ensure the quality of published research via the process of peer review and revision, formal statistical review and editorial recommendations about wording, data presentation and graphics. Additional "mechanisms to improve transparency, trial reporting and overall study quality" include clinical trial registration and adherence to defined research guideline checklists. ${ }^{1}$ In this issue of Heart, Sims and colleagues ${ }^{1}$ looked at 19 major cardiology journals' author instructions for recommendations about trial registration and research guidelines. They then looked at articles in those journals to see if the trial registration number was indicated in the final publication (figure 1). Overall, 42\% of journals did not require trial registration and $37 \%$ did not recommend even a single reporting guideline. These findings suggest that more attention to trial registration and research guideline checklists is needed by other major cardiology journals. Heart does require submission of the appropriate research checklist, lists the most common guidelines and directs authors to the Equator Network website (http://www.equator-network.org/) when further information is needed. All cardiovascular researchers should be familiar with these checklists and use them in study design (not just when writing the paper), regardless of which journal publishes the final paper.

Prognosis in adults with Eisenmenger syndrome (ES) is very poor and we had few therapeutic options until about 10 years ago when advanced pulmonary

Correspondence to Professor Catherine M Otto, Division of Cardiology, University of Washington, Seattle, WA 98195, USA; cmotto@uw.edu

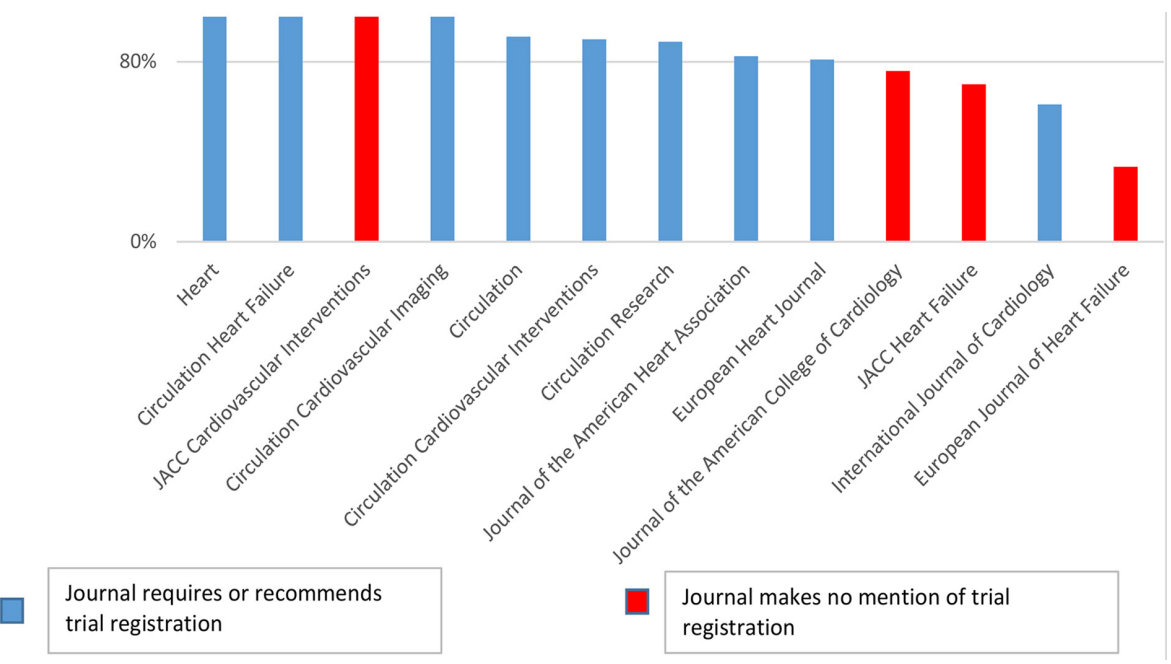

Figure 1 Frequency of published trial registry number.

vasodilator therapy (AT) was shown to be safe and improve exercise capacity. However there still is little data on any potential survival benefit of AT and whether treatment effects are modulated by associated clinical factors. In an observational study of 253 patients with ES in Australia and New Zealand, Arnott and colleagues $^{2}$ found a lower risk of death or heart transplantation at 1 year in those treated with AT compared with those who never received AT (mortality rate of 4.8 vs $8.4 \%$ per year), even though patients receiving AT had more severe

symptoms at baseline. Multivariable analysis showed that exposure to AT was independently associated with greater survival (figure 2).

In an accompanying editorial, $\mathrm{Tay}^{3}$ provides detailed tables summarising the three previous observational studies on AT for ES to put this new data in context. The current study by Arnott and colleagues has the largest proportion of patients receiving AT, the longest duration of follow-up (mean 8.3 years) and shows the benefit of AT in the sickest patients. He emphasises: "The success

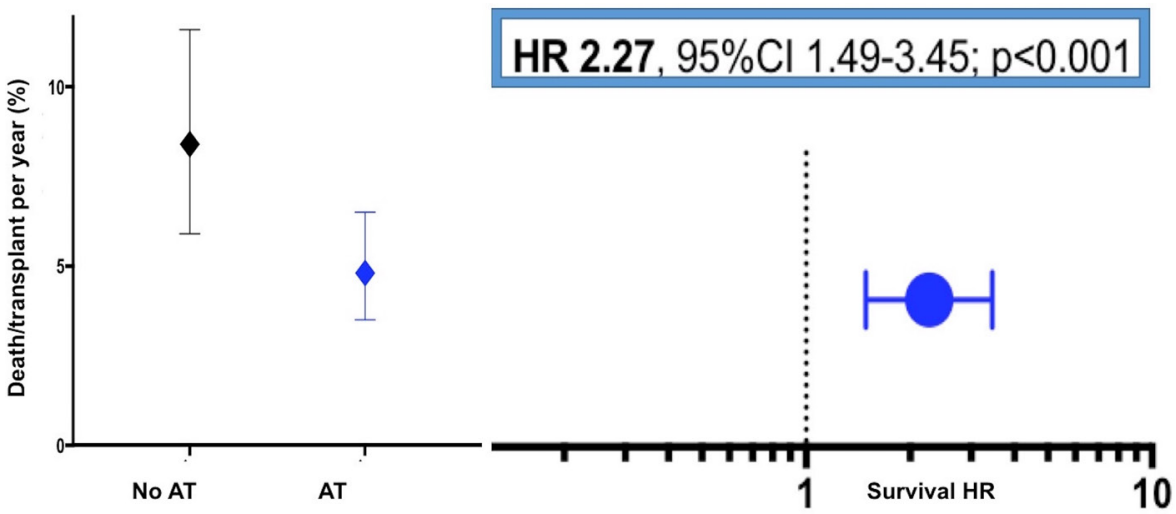

Figure 2 Greater survival in those exposed to advanced therapy. The left panel depicts the rate of death/transplant per person year in \% for those exposed to advanced therapy and those never exposed. Error bars represent $95 \% \mathrm{Cl}$. The right panel depicts the survival HR for those exposed to advanced therapy based on Cox multivariable analysis. AT, exposure to advanced therapy during study; no AT, no exposure to advanced therapy. 


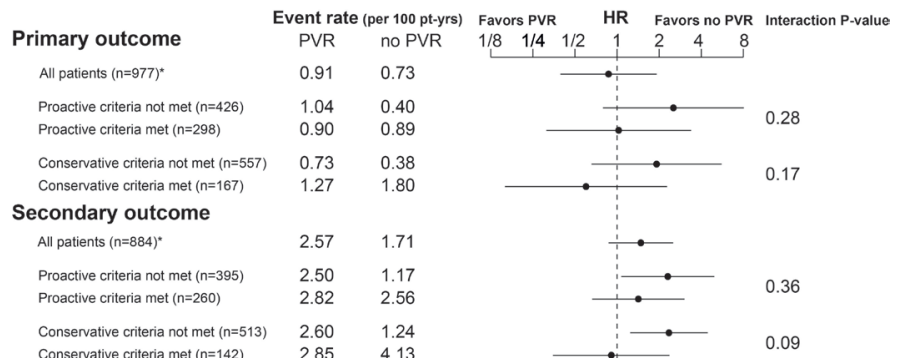

Figure 3 Event rate according to proactive and conservative criteria and pulmonary valve replacement (PVR) status. Event rates according to prespecified PVR and proactive/conservative criteria subgroups. *The PVR propensity-corrected HR of event (PVR compared with no PVR) for all patients is displayed as the overall estimate for the primary and secondary outcome. The unadjusted HR of event within subgroups is also displayed in this figure including the interaction test $p$ value.
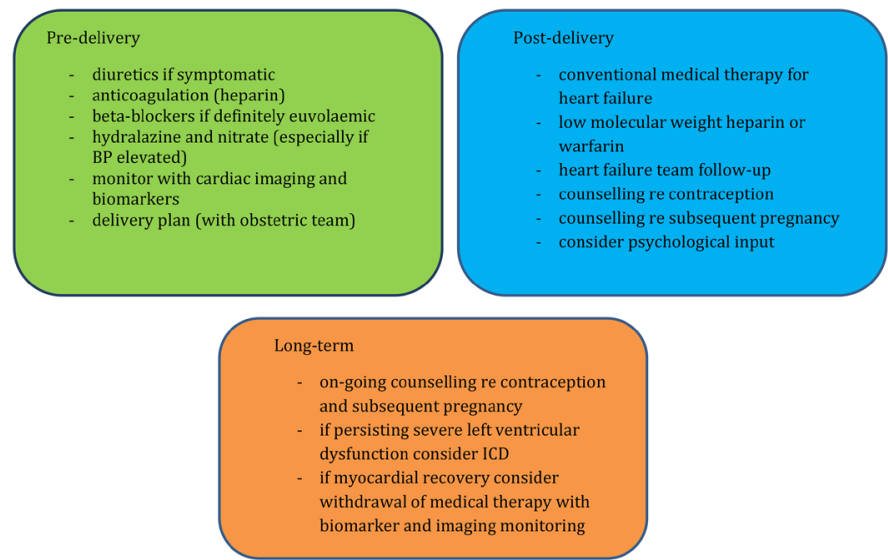

Figure 4 Peripartum cardiomyopathy management. BP, blood pressure; ICD, implantable cardioverter defibrillator.

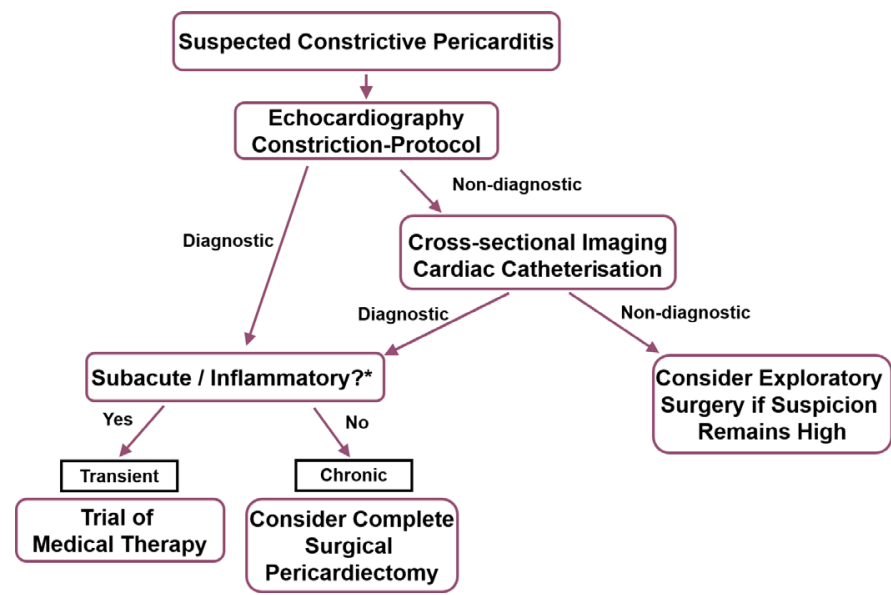

Figure 5 Simplified diagnostic algorithm for the diagnosis and treatment of symptomatic constrictive pericarditis. *Based on time course and clinical assessment that may include CMR MRI and inflammatory markers.

of this study rested partially on good coordinated systems in healthcare. The strictly mandated national level therapeutic guidelines had to be adhered to, leading to a more homogenised delivery of care while a good national registry of deaths enabled accurate mortality data to be collected." In the future, "Randomised controlled trials should begin steering away from conventional surrogates of outcomes (eg, 6 min walk test) but focus more on harder endpoints like mortality and morbidity outcomes like in pulmonary arterial hypertension trials.”

Adults patients with a repaired tetralogy of Fallot (rTOF) often have significant pulmonic valve dysfunction, prompting consideration of pulmonic valve replacement (PVR). In a series of 977 rTOF patients, Bokma and colleagues report that the $45 \%$ of patients who had undergone PVR did not have a lower mortality or incidence of sustained ventricular tachycardia over an average follow-up of 5.3 years compared with rTOF patients without a PVR. ${ }^{4}$ In patients who did not meet consensus criteria for PVR but still had the procedure, there were more adverse events after PVR compared with patients treated (appropriately) without PVR (figure 3).

Latus and Tutarel ${ }^{5}$ comment in response to this data that: "The reported 'mid-term' outcomes shed a critical light on several aspects of our current theory regarding the time course and underlying mechanisms of right ventricular(RV) failure, arrhythmias and sudden cardiac death. If PVR seems not to improve outcome or even increases risk when performed 'too early', our hypothesis of an association between RV regression and improved outcome following PVR seems error prone." They suggest that, in addition to severity of pulmonic regurgitation and RV dilation, we might also need to consider focal fibrosis and surgical scar as possible risk factors for ventricular tachycardia in rTOF patients. Perhaps risk will be lower with transcatheter, compared with surgical PVR approaches, although questions remain about valve durability and susceptibility to endocarditis.

The Education in Heart article in this issue addresses the pathophysiology, diagnosis and management of peripartum cardiomyopathy. ${ }^{6}$ Key points are to: (1) consider the diagnosis in women presenting with signs of heart failure during pregnancy to up to several months after delivery, (2) make the diagnosis with serum markers and echocardiography and (3) remember that many patients recover function within 12 months on conventional heart failure therapy with a mortality rate now $<10 \%$ at 1 year. However, there is a risk of recurrence with future pregnancieslong term management should involve an interdisciplinary team with patient counselling about contraception and longterm medical therapy for heart failure (figure 4).

A state-of-the-art review article on constrictive pericarditis by Welch ${ }^{7}$ is an invaluable guide to this uncommon, 
but devastating, clinical condition. Diagnosis is challenging, often delayed, and management options are not ideal (figure 5).

Competing interests None declared.

Patient consent Not required.

Provenance and peer review Commissioned; internally peer reviewed.

(C) Article author(s) (or their employer(s) unless otherwise stated in the text of the article) 2018. All rights reserved. No commercial use is permitted unless otherwise expressly granted.

\section{(D) Check for updates}

To cite Otto CM. Heart 2018;104:707-709.

Heart 2018;104:707-709.

doi:10.1136/heartjnl-2018-313339

\section{REFERENCES}

1 Sims MT, Bowers AM, Fernan JM, et al. Trial registration and adherence to reporting guidelines in cardiovascular journals. Heart 2018;104:753-9.

2 Arnott C, Strange G, Bullock A, et al. Pulmonary vasodilator therapy is associated with greater survival in Eisenmenger syndrome. Heart 2018;104:732-7.
3 Tay ELW. Managing the Eisenmenger syndrome: a call to arms. Heart 2018;104:714-6.

4 Bokma JP, Geva T, Sleeper LA, et al. A propensity scoreadjusted analysis of clinical outcomes after pulmonary valve replacement in tetralogy of Fallot. Heart 2018;104:738-44.

5 Latus $\mathrm{H}$, Tutarel 0 . Outcomes after pulmonary valve replacement in patients with repaired tetralogy of Fallot: end of a success story? Heart 2018;104:717-8.

6 Jackson AM, Dalzell JR, Walker NL, et al. Peripartum cardiomyopathy: diagnosis and management. Heart 2018;104:779-86.

7 Welch TD. Constrictive pericarditis: diagnosis, management and clinical outcomes. Heart 2018;104:725-31. 